www.nature.com/ejhg

\title{
Systematic analysis of the regulatory and essential myosin light chain genes: genetic variants and mutations in hypertrophic cardiomyopathy
}

\author{
Zhyldyz T Kabaeva ${ }^{1,2}$, Andreas Perrot ${ }^{1}$, Bastian Wolter ${ }^{1}$, Rainer Dietz ${ }^{1}$, Nuno Cardim ${ }^{3}$, \\ João Martins Correia ${ }^{3}$, Hagen D Schulte ${ }^{4}$, Almaz A Aldashev ${ }^{2}$, Mirsaid M Mirrakhimov ${ }^{2}$ \\ and Karl Josef Osterziel ${ }^{\star, 1}$
}

\begin{abstract}
${ }^{1}$ Charité/Kardiologie, Campus Buch und Virchow-Klinikum, Humboldt-Universität zu Berlin, Germany; ${ }^{2}$ National Center of Cardiology and Internal Medicine, Bishkek, Kyrgyzstan; ${ }^{3}$ Cardiology Department of Hospital Pulido Valente, Lisbon, Portugal; ${ }^{4}$ Klinik für Thorax- und kardiovaskuläre Chirurgie, Heinrich-Heine-Universität, Düsseldorf, Germany
\end{abstract}

Hypertrophic cardiomyopathy (HCM) can be caused by mutations in genes encoding for the ventricular myosin essential and regulatory light chains. In contrast to other HCM disease genes, only a few studies describing disease-associated mutations in the myosin light chain genes have been published. Therefore, we aimed to conduct a systematic screening for mutations in the ventricular myosin light chain genes in a group of clinically well-characterised HCM patients. Further, we assessed whether the detected mutations are associated with malignant or benign phenotype in the respective families. We analysed 186 unrelated individuals with HCM for the human ventricular myosin regulatory (MYL2) and essential light chain genes (MYL3) using polymerase chain reaction, single strand conformation polymorphism analysis and automated sequencing. We found eight single nucleotide polymorphisms in exonic and adjacent intronic regions of MYL2 and MYL3. Two MYL2 missense mutations were identified in two Caucasian families while no mutation was found in MYL3. The mutation Glu22Lys was associated with moderate septal hypertrophy, a late onset of clinical manifestation, and benign disease course and prognosis. The mutation Arg58GIn showed also moderate septal hypertrophy, but, in contrast, it was associated with an early onset of clinical manifestation and premature sudden cardiac death. In conclusion, myosin light chain mutations are a very rare cause of HCM responsible for about $1 \%$ of cases. Mutations in MYL2 could be associated with both benign and malignant HCM phenotype. European Journal of Human Genetics (2002) 10, 741 - 748. doi:10.1038/sj.ejhg.5200872

Keywords: cardiomyopathy; myosin light chains; hypertrophy; sudden cardiac death; genetics

\section{Introduction}

Hypertrophic cardiomyopathy is a heart disorder characterised by left and/or right ventricular hypertrophy, which is usually asymmetric and involves the interventricular septum. ${ }^{1}$ The prevalence of HCM in the general population is believed to be $0.2 \% .^{2}$ The clinical expression of hypertro-

*Correspondence: KJ Osterziel, Charité/Franz-Volhard-Klinik, Wiltbergstr. 50; 13125 Berlin; Germany. Tel: ++49-30-94172501;

Fax: ++49-30-94172279; E-mail: osterziel@fvk-berlin.de

Received 21 March 2002; revised 27 June 2002; accepted 10 July 2002 phy, course of the disease and prognosis of the patients show wide heterogeneity. In the majority of patients the disease is benign, but some patients show a high incidence of sudden death. ${ }^{3,4}$ Some of the identified causal mutations have been shown to be associated with a poor prognosis of HCM and a high incidence of sudden cardiac death. ${ }^{5}$

The majority of HCM is familial with an autosomal dominant pattern of inheritance. Mutations in 10 different genes all encoding for sarcomeric proteins have been shown to cause the disease. ${ }^{6}$ In addition to this locus heterogeneity, there is a wide allelic heterogeneity; about 100 different 
mutations have been identified in these disease genes. ${ }^{7}$ Only a few families with mutations in the ventricular myosin essential (ELC) and regulatory (RLC) light chain genes have been reported so far worldwide. ${ }^{8-11}$ Therefore, it is difficult to get reliable data for genotype/phenotype correlations in these HCM disease genes.

The RLC is encoded by the MYL2 gene located on chromosome $12 \mathrm{q} 23-\mathrm{q} 24.3$ and consists of 166 amino acids. ${ }^{12}$ The ELC composed of 194 amino acids is encoded by the MYL3 gene mapped to chromosome $3 \mathrm{p} .{ }^{13}$ Myosin consists of two heavy chains and two pairs of light chains. The light chains stabilise the $\alpha$-helical neck region of myosin and are located in close proximity to the myosin ATP binding and actin binding domains. ${ }^{14,15}$ Apart from the structural role, ELC and RLC are assumed to modulate and regulate actinmyosin interaction in striated muscle. ${ }^{16,17}$

We conducted a systematic screening for mutations in the ventricular myosin light chain genes in a group of 186 clinically well-characterised HCM patients. We found a number of genetic variants in MYL2 and in MYL3 as well as two mutations in MYL2 associated with HCM in two families. Further, we showed that mutations in MYL2 are associated with either benign or malignant HCM phenotype depending on the respective mutation.

\section{Methods}

\section{Clinical evaluation}

Informed consent was obtained in accordance with the guidelines of the ethical commission of the Charité/FranzVolhard-Klinik Berlin, the National Center for Cardiology and Internal Medicine Bishkek, Hospital Pulido Valente Lisbon, and Klinik für Thorax- und kardiovaskuläre Chirurgie Düsseldorf. Clinical evaluation was performed and blood samples were drawn from 186 unrelated patients with HCM, 14 family members, and 210 control individuals. All patients were evaluated on the basis of medical history, physical examination, 12-lead electrocardiogram, M/Bmode and Doppler echocardiography, and, in some cases,
Holter electrocardiography (Holter ECG), heart catheterisation, and cardiac magnetic resonance imaging. The echocardiographic evaluation was performed without knowledge of genetic results according to guidelines of the American Society of Echocardiography. ${ }^{18}$ Left ventricular (LV) wall thickness of $\geqslant 13 \mathrm{~mm}^{19}$ was used as the inclusion criterion in the absence of other known causes for LV hypertrophy (eg hypertension, aortic stenosis, etc). In family members, LV wall thickness was evaluated in relation to age, weight and body surface area according to Henry et al. ${ }^{20}$

\section{Genetic screening}

Genomic DNA was isolated by standard techniques. Seven coding exons of MYL2 and six coding exons of MYL3 were amplified using polymerase chain reaction. Oligonucleotide primers were designed in the exon flanking intronic regions on the basis of reference sequences from GenBank (Table 1). Reference genomic DNA sequence accession numbers are L01652 and J04462 for MYL2 and MYL3, respectively; reference cDNA sequence accession numbers are X66141 and M24122 for MYL2 and MYL3, respectively.

Mutation screening was performed by single strand conformation polymorphism analysis (SSCP) under two different conditions with respect to gel composition and running temperature. Bands were vizualised by silver staining according to the standard protocol (Pharmacia, Sweden). Samples with aberrant band patterns were subjected to automated sequencing of both strands using dye primer chemistry on a 373 DNA sequencing system (Applied Biosystems, USA).

Screening for mutation Gly22Lys in MYL2 within family $\mathrm{K}$ and controls was done by restriction enzyme digestion with Taq I (New England Biolabs, USA). Restriction enzyme Sty I (New England Biolabs, USA) was used to confirm the 169C $>$ G sequence variant in MYL2.

Statistical analysis was performed using software StatView (Abacus Concepts Inc, Berkeley, California, USA).

Table 1 Oligonucleotide primers used to amplify coding exons of MYL2 and MYL3

\begin{tabular}{|c|c|c|c|}
\hline Gene & Exon & Forward primer & Reverse primer \\
\hline \multirow[t]{8}{*}{ MYL2 } & 1 & 5'-АССТАTGACTGC CAAAAGCG-3' & 5'-GTAGTGGCTTCCTCTCCTCG-3' \\
\hline & 2 & 5'-GGGGC CTGAC CTAGTTTTTT-3' & 5'-TTTGGGATTGTTTGGAGGAT-3' \\
\hline & 3 & 5'-TCСАCTCCTGCCAACTCCTT-3' & 5'-ACCCACCTCCTGCTCCTCAT-3' \\
\hline & 4 & 5'-GCСTCATCAСCCCATCTCTG-3' & 5'-AGCCCCCCCGAAGAAACATA-3' \\
\hline & 5 & 5'-TCATCTCTGGGGGAACTTGG-3' & 5'-TGTGTGTGTGTAGGGGGGAC-3' \\
\hline & 6 & 5'-AAAGGGGTGCTGAAGGCTGA-3' & 5'-AGACGAGAGGGGAGACGGAG-3' \\
\hline & $7(\mathrm{~A})$ & 5'-TCCGTCTCAGTTCCCCTCCC-3' & 5'-GTACCCATAGCCACCCAGGC-3' \\
\hline & $7(B)$ & 5'-GCCCCATTTATCCACCTCCA-3' & 5'-GGCTTTGGTCATCCAGGTAA-3' \\
\hline \multirow[t]{6}{*}{ MYL3 } & 1 & 5'-GGGGTCATGAGGTATCCGGG-3' & 5'-TCСАСТСАСTTGCCCTGCTC-3' \\
\hline & 2 & 5'-C CACCTTTTAAGCCGGGCAT-3' & 5'-CCGCAGGACATCCCCACACT-3' \\
\hline & 3 & 5'-ATTGAAGGTGAGCAGGGGTC-3' & 5'-TAACACTATGGGGGCTCTCG-3' \\
\hline & 4 & 5'-GTGTGAGAGGTGGGGATAGC-3' & 5'-TGGAAGGAGTTGGGGTAGGG-3' \\
\hline & 5 & 5'-TGACTCAGCCTCCCACTCCT-3' & 5'-CTCCCCTCC CAGAAGACCCC-3' \\
\hline & 6 & 5'-GGTCTTCTGGGAGGGGAGTG-3' & 5'-TTCCCTGGGCTTC CTGAGAG-3' \\
\hline
\end{tabular}




\section{Results}

A total of 186 unrelated HCM patients including 119 males and 67 females aged 14-85 were examined for genetic variants in MYL2 and MYL3. Clinical data of these patients are summarized in Table 2.

\section{Genetic variants in the human MYL2 and MYL3 genes}

Eight single nucleotide polymorphisms (SNP) were identified in MYL2 and MYL3. Furthermore, a number of differences were identified compared to the reference sequences (see overview in Figure 1).

Three rare SNPs, which did not change the amino acid, were detected in coding regions. Each of these SNPs was identified only in one individual out of 186 patients. The $132 \mathrm{~T}>\mathrm{C}$ (Ile44Ile) substitution was detected in exon 3 of MYL2. The other two substitutions, 69C $>\mathrm{T}$ (Pro23Pro) and $420 \mathrm{C}>\mathrm{T}$ (Phe140Phe), were identified in MYL3 in exons 1 and 4, respectively. Five more frequent SNPs were located in the exon flanking regions of introns 4 and 5 of MYL2 (three nucleotide substitutions, one insertion and one deletion). We detected no intronic SNP in MYL3.

Compared to the reference sequences of MYL2 and MYL3, we detected 24 nucleotide differences. These 24 nucleotide differences were considered as differences from the reference sequence rather than polymorphisms because they were present in all patient and control samples sequenced by us. The differences from the MYL2 reference genomic sequence were present mostly as nucleotide substitutions $(n=10)$. In MYL3 they were present mostly as nucleotide insertions $(n=7)$. Four single nucleotide deletions and two insertions were detected in the MYL2 sequence. All differences in both genes were located in noncoding regions apart from variants $240 \mathrm{~A}>\mathrm{T}$ (exon 4) and $169 \mathrm{C}>\mathrm{G}$ (exon 3) in MYL2. An interesting finding is the $169 \mathrm{C}>\mathrm{G}$ variant located at the donor site of exon 3 . Furthermore, we detected the lack of adenine and guanine in the acceptor splice region of exon 4 and found another adenine-guanine splice site three nucleotides upstream. Therefore, the acceptor splice site of exon 4 was shifted three nucleotides upstream (see Figure 2A). This finding was in agreement with the reference cDNA sequence and was confirmed by J Flavigny, Paris (personal communication).

\section{Missense mutations in human MYL2}

We identified two missense mutations, Glu22Lys and Arg58Gln, in human MYL2 in two unrelated individuals out of 186 HCM patients examined (Figure 1).

The mutation Glu22Lys was initially observed in proband II-3 of family K who showed a clear SSCP abnormality in exon 2 of MYL2. DNA sequencing revealed a heterozygous G-to-A substitution at nucleotide 64 resulting in a replacement of glutamic acid by lysine (Figure 2). This base change caused a loss of one of two normal Taq I restriction sites in the exon 2 amplimer allowing its independent
Table 2 Clinical features of HCM patients screened in the present study

\begin{tabular}{|c|c|}
\hline Number of patients (n) & 186 \\
\hline Age, years & $55.1 \pm 15.5$ \\
\hline \multicolumn{2}{|l|}{ Sex, \% (n) } \\
\hline Male & $64.0(119)$ \\
\hline Female & $35.0(67)$ \\
\hline Age at diagnosis, years & $46.0 \pm 16.9$ \\
\hline \multicolumn{2}{|l|}{ NYHA class, $\%(n)$} \\
\hline 1 & $29.0(54)$ \\
\hline II & $52.7(98)$ \\
\hline III & $17.2(32)$ \\
\hline IV & $1.1(2)$ \\
\hline Morrow myectomy, \% (n) & $23.4(44)$ \\
\hline Nonsurgical septal reduction, \% (n) & $2.1(4)$ \\
\hline \multicolumn{2}{|l|}{ Rhythm, \% (n) } \\
\hline Sinus & $90.3(168)$ \\
\hline Atrial fibrillation & $7.5(14)$ \\
\hline Pacemaker & $2.2(4)$ \\
\hline \multicolumn{2}{|l|}{ Heart block, \% (n) } \\
\hline LAH & $11.9(22)$ \\
\hline RBBB/Incomplete RBBB & $8.1(15)$ \\
\hline LBBB & $5.4(10)$ \\
\hline Others* & $4.9(9)$ \\
\hline Negative T waves, \% ( $n)$ & $51.1(95)$ \\
\hline Abnormal Q waves, \% (n) & $38.7(72)$ \\
\hline IVS thickness, mm & $19.9 \pm 5.0$ \\
\hline PW thickness, mm & $12.2 \pm 3.1$ \\
\hline IVS/PW & $1.8 \pm 2.0$ \\
\hline LVEDD, $\mathrm{mm}$ & $45.7 \pm 6.0$ \\
\hline SAM, \% $(n)$ & $61.3(114)$ \\
\hline LVOT gradient increased, \% (n) & $65.1(121)$ \\
\hline \multicolumn{2}{|l|}{ Maron type, \% (n) } \\
\hline I & $23.1(43)$ \\
\hline II & $38.7(72)$ \\
\hline III & $30.1(56)$ \\
\hline IV & $8.1(15)$ \\
\hline
\end{tabular}

Data are expressed as mean \pm standard deviation or as relative (\%) and absolute (n) values. *Atrioventricular block $\mathrm{III}^{\circ}$, bifascicular block and posterior fascicular block. $L A H$, anterior fascicular block; RBBB, right bundle branch block; LBBB, left bundle branch block; IVS, interventricular septum; PW, left ventricular posterior wall; LVEDD, left ventricular end-diastolic dimension; SAM, systolic anterior motion of mitral valve; LVOT, left ventricular outflow tract.

confirmation by restriction enzyme digestion. The same Glu22Lys mutation was detected in further six individuals of family K (II-5, III-2, III-3, III-5, IV-1, and IV-2) (Figure $3)$. Two hundred and ten control alleles failed to show this mutation. Within genetically affected members of family $\mathrm{K}$, four individuals (II-3, III-5, IV-1, and IV-2) had apparent HCM (Table 3 ). The phenotype of the remaining individuals II-5, III-2, and III-3 was defined as 'uncertain': individuals II-5 and III- 2 did not fulfil the diagnostic criteria of HCM while in the case of individual III-3 only ECG but no echo data were available. The members of family $\mathrm{K}$ had mild to moderate septal hypertrophy and no outflow tract obstruction. All genetically affected individuals were asymptomatic, apart from proband II-3 and his sister II-5, 


\section{A) MYL2, the ventricular myosin regulatory light chain gene}

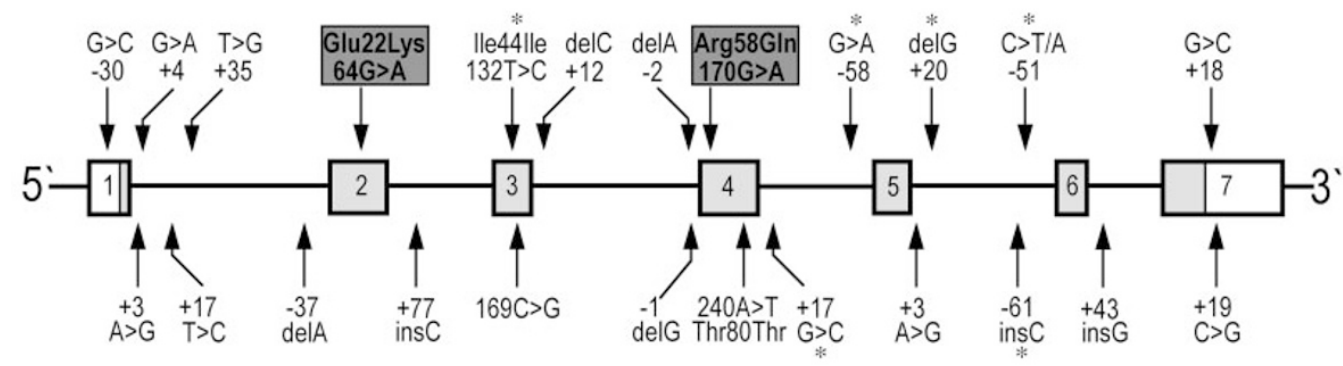

B) MYL3, the ventricular myosin essential light chain gene

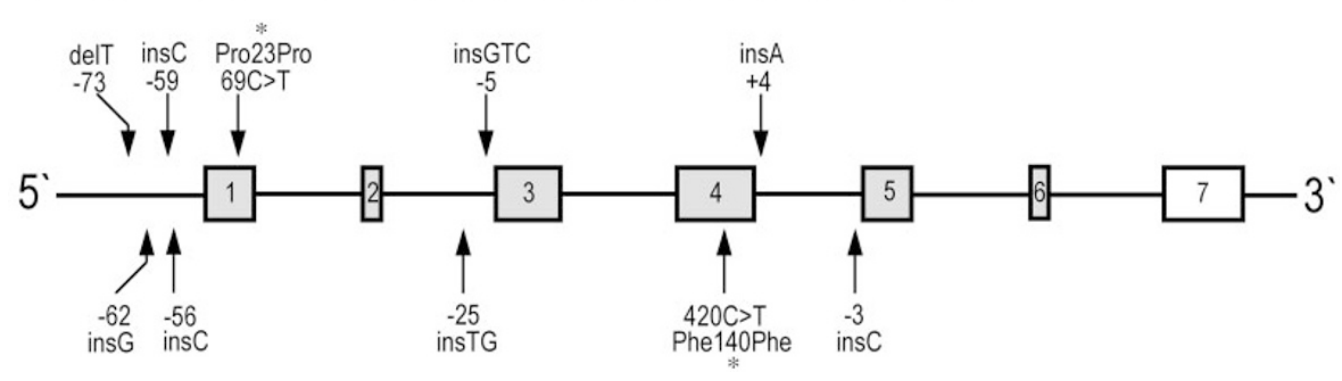

Figure 1 Schematic representation of the ventricular myosin regulatory light chain gene (MYL2) and the ventricular myosin essential light chain gene (MYL3). Numbering was performed according to Beaudet et al. ${ }^{21}$ Boxes represent exons, light shaded boxes represent coding DNA of the gene; mutations found in this study are dark shaded; variants with asterisk indicate nucleotide polymorphisms; variants without asterisk indicate difference from the reference genomic DNA sequence. (A) Location of the nucleotide variants and two mutations of MYL2. Reference genomic DNA and CDNA accession numbers are L01652 and X66141 (GenBank), respectively. (B) Location of the nucleotide variants found in MYL3. Reference genomic DNA and CDNA accession numbers are J04462 and M24122 (GenBank), respectively.

who reported periodic chest pain and dyspnea; proband II-3 had also reported palpitations.

The mutation Arg58Gln was initially identified in proband II-2 of family B who showed an aberrant band pattern on SSCP analysis of exon 4 of MYL2. DNA sequencing revealed a heterozygous G-to-A transition at nucleotide 173 causing a change of arginine to glutamine at codon 58 (Figure 2). The Arg58Gln mutation was not observed in 210 control alleles subjected to SSCP analysis. The mutation was not found in proband's mother I-2 who showed normal cardiac findings (Figure 3). In two other family members, proband's father I-1 and sister II-1, the genetic analysis could not be performed, because they died and no DNA could be obtained. The proband of family $\mathrm{B}$ had nonobstructive HCM first diagnosed at the age of 7 years. Clinical symptoms included premature fatigue on exertion, palpitations, and dizziness. Because of observation of ventricular tachycardia degenerating into ventricular fibrillation on electrophysiological examination, the proband underwent an implantation of a cardioverter defibrillator at the age of 25. She also had recurrent events of supraventricular tachycardia. Echocardiography revealed asymmetric septal hypertrophy extending to the LV apex and lateral free wall (Table 3). Her sister, who suffered from asymmetric obstructive HCM, died suddenly at the age of 21. Also her father died unexpectedly at young age; HCM was found on autopsy.

\section{Discussion}

We screened a group 186 unrelated HCM patients and identified genetic variants in both human ventricular myosin light chain genes. Two mutations in the RLC gene (MYL2) were found in two Caucasian families while no mutation was detected in the ELC gene (MYL3). Further, we showed that mutations in MYL2 are associated with either benign or malignant HCM phenotype.

The identification of a large number of genetic variants showed the high sensitivity and accuracy of our screening method. Sequencing confirmed all aberrant band patterns detected by SSCP. Therefore, we believe that we might have missed possibly only few variants in these genes. Other studies showed that sensitivities of about $90 \%$ can be achieved using SSCP as a screening method. ${ }^{22}$ Eight SNPs were identified in intronic and exonic regions of MYL2 and MYL3. The exon flanking regions of introns 4 and 5 of MYL2 seem to be highly polymorphic since five of the 


\section{A) Control}

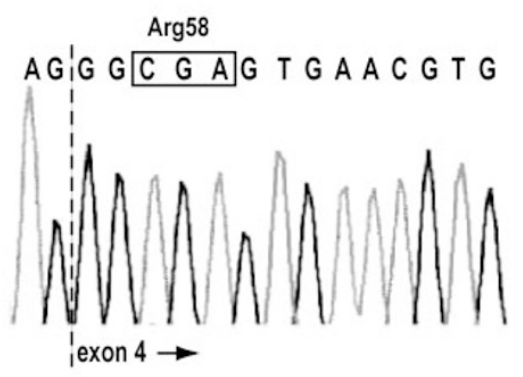

\section{Proband of family B}

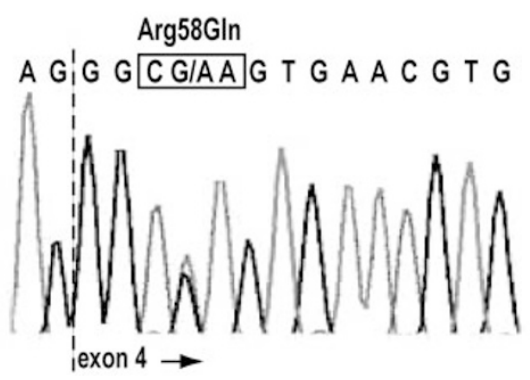

\section{B) Control}

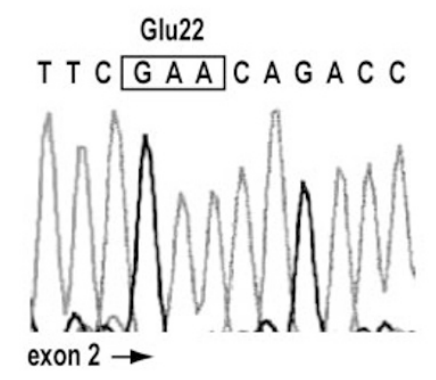

Proband of family K

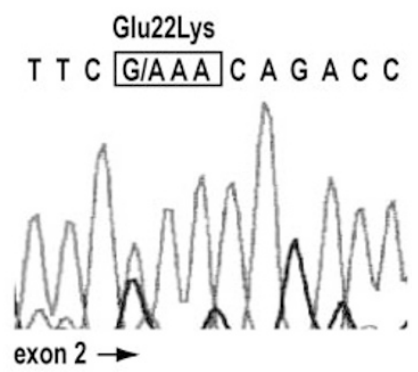

Figure 2 Electropherograms of MYL2 of the probands and controls. (A) Electropherograms of corresponding regions of exon 2 of a control individual and the proband of family B. Sequence of the proband of family B showed a heterozygous G-to-A substitution resulting in an exchange of arginine to glutamine at codon 58. (B) A heterozygous G-to-A substitution in the proband of family $\mathrm{K}$ caused a change of glutamic acid to lysine at codon 22.

eight SNPs were located there. Further studies are needed to estimate the frequency of the identified SNPs in patient cohorts and in the general population and whether they have a potential functional implication for the disease. The 24 differences from the reference sequences detected by us underline how frequent sequencing errors occur and how careful one should be to use reference sequences. Missense mutations in MYL2 were present in about $1 \%$ of examined HCM cases whereas no mutation was identified in MYL3. This indicates that the frequency of mutations in these genes seems to be lower than estimated by Flavigny et al. (with 7\%) and Andersen et al. (with 4.4\%). In contrast to other HCM disease genes, only four studies on ELC/RLC mutations have been published. ${ }^{8-11}$ Consequently, detailed phenotypic data on families with myosin light chain mutations remain as rare as mutations in these genes; very little is known about disease course and prognosis. Especially in this rare form of HCM, every single family identified is valuable. The data presented in this work show that clinical phenotypes associated with myosin light chain mutations differ markedly.

The Glu22Lys mutation in MYL2 cosegregated with HCM in family $\mathrm{K}$ across three generations with affecteds in each generation. This is the largest family with this mutation reported until now. Poetter et al. ${ }^{8}$, who identified two brothers and one unrelated individual with the Glu22Lys mutation, described briefly a phenotype with massive hypertrophy of the cardiac papillary muscles and adjacent ventricular tissue causing midcavity obstruction but presented no data about course and prognosis. In our study, none of the individuals bearing the Glu22Lys mutation had massive midventricular hypertrophy. Therefore, this phenotype seems not to be specific for patients with mutations in RLC, as it has been also proposed by a recent study. ${ }^{11}$ Further, we observed that the Glu22Lys mutation was associated with favourable disease course and prognosis. There was no case of sudden cardiac death in the family and most of the affected family members had only mild hypertrophy, late onset of symptoms or no symptoms at all.

In contrast, the Arg58Gln mutation in MYL2 identified in family B was associated with two cases of premature sudden cardiac death. Although we could not perform genetic analysis on these two deceased individuals they could be considered as obligate carriers of this mutation. Further, the mutation was associated with symptoms manifesting in early childhood as premature fatigue and later as 
A) Family B

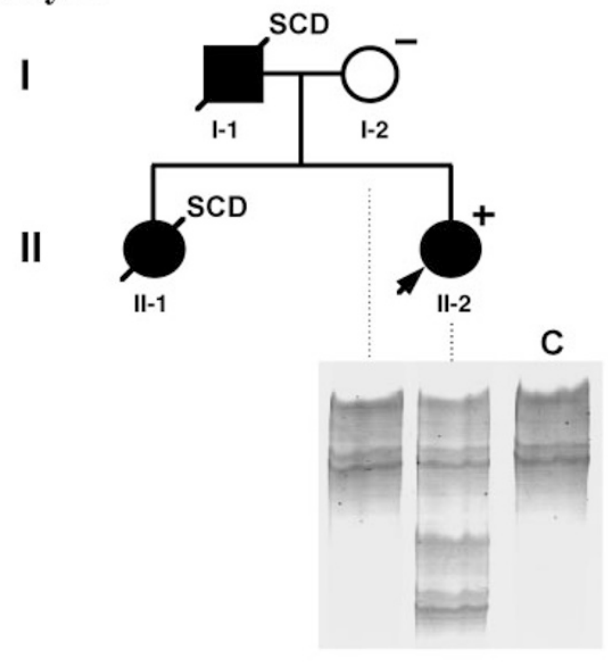

B) Family $\mathrm{K}$

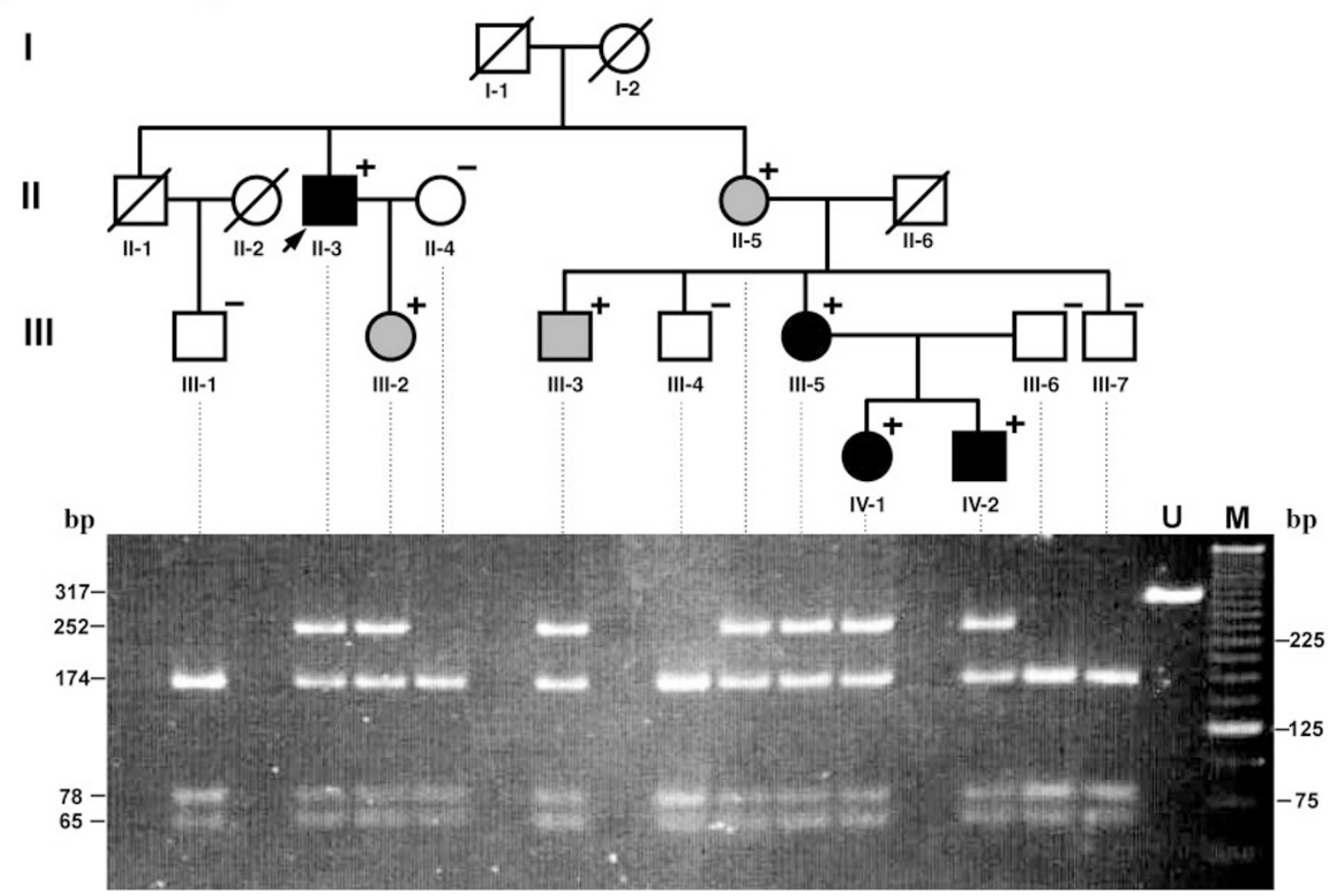

Figure 3 Pedigrees and results of genetic analysis of families B and K. Black symbols represent clinically affected patients; white symbols, clinically unaffected individuals; grey symbols, individuals with uncertain phenotype; symbols with plus sign above, genetically affected individuals; symbols with minus sign above, genetically unaffected individuals; and symbols with diagonal slash, decreased individuals. Probands are indicated by arrows. SCD, sudden cardiac death. (A) Pedigree of family B and results of SSCP analysis. The proband II-2 showed an aberrant band pattern absent in her mother I-2 and control C. (B) Pedigree of family $\mathrm{K}$ with a picture of a $4 \%$ agarose gel containing restriction digests below. Lane $U$ contains an undigested 317 base pair (bp) PCR product of exon 2 of MYL2. Lane M contains $125 \mathrm{bp}$ DNA ladder with sizes of bands shown on the right side of the gel picture. Sizes of digestion products are shown on the left side of the gel picture. Two normal $T a q^{\alpha}$ I restriction enzyme sites produce fragments of 174,78 and 65 bp that are present in all family members. A heterozygous G-to-A transition removes one of the two Taq ${ }^{\alpha}$ I restriction sites resulting in fragments of 252 and 65 bp. The 252 bp fragment is present in genetically affected family members II-3, III-2, III-3, II-5, III-5, IV-1, IV-2. 
Table 3 Clinical features of genetically affected individuals of the families

\begin{tabular}{|c|c|c|c|c|c|c|c|c|c|c|c|c|c|c|c|c|}
\hline $\begin{array}{l}\text { Pedigree } \\
\text { number }\end{array}$ & Mutation & $\begin{array}{c}\text { Age } \\
\text { (years) }\end{array}$ & $\begin{array}{c}\text { Age } \\
\text { diagn. } \\
\text { (years) }\end{array}$ & $\begin{array}{l}B S A \\
\left(m^{2}\right)\end{array}$ & $\begin{array}{l}\text { Weight } \\
(\mathrm{kg})\end{array}$ & $\begin{array}{l}\text { Heart } \\
\text { block }\end{array}$ & $\begin{array}{c}\mathrm{Neg} \\
T\end{array}$ & $\begin{array}{c}A b n \\
Q\end{array}$ & $\begin{array}{c}S-L \\
\text { index } \\
(m V)\end{array}$ & $\begin{array}{c}\text { IVS } \\
(\mathrm{mm})\end{array}$ & $\begin{array}{c}P W \\
(\mathrm{~mm})\end{array}$ & $\begin{array}{l}\text { IVS/ } \\
P W\end{array}$ & $\begin{array}{c}\text { LVEDD } \\
(\mathrm{mm})\end{array}$ & $\begin{array}{l}\text { Maron } \\
\text { type }\end{array}$ & $\begin{array}{c}\text { NYHA } \\
\text { class }\end{array}$ & $\begin{array}{l}\text { Clinical } \\
\text { Status }\end{array}$ \\
\hline \multicolumn{17}{|l|}{ Family K } \\
\hline II-5 & Glu22Lys & 62 & 62 & 2.45 & 139 & no & no & no & 1.9 & $13^{*}$ & n.d. & n.a. & 47 & 1 & II & uncertain \\
\hline III-2 & Glu22Lys & 33 & 33 & 2.08 & 98 & no & no & yes & 2.4 & 10 & 7 & 1.4 & 48 & n.a. & 1 & uncertain \\
\hline III-5 & Glu22Lys & 42 & 42 & 2.06 & 96 & no & no & yes & 3.1 & 15 & 8 & 1.8 & 53 & 1 & I & affected \\
\hline \multicolumn{17}{|l|}{ Family B } \\
\hline $\mathrm{II}-2$ & Arg58GIn & 27 & 7 & 1.58 & 56 & no & yes & no & 4.8 & 21 & 7 & 3 & 39 & III & II & affected \\
\hline
\end{tabular}

BSA, body surface area; neg T, negative T waves; abn Q, abnormal Q waves; S-L index, Sokolow-Lyon index; IVS, interventricular septum; PW, left ventricular posterior wall; LVED, left ventricular end-diastolic dimension; LBBB, left ventricular bundle branch block; NYHA, New York Heart Association; n.d., not determined; n.a., not applicable. *This value of the IVS thickness was normal in relation to BSA of individual II-5. ${ }^{* *}$ This value of the IVS thickness was increased in relation to BSA of individual IV-1.

arrhythmias. The pattern of LV hypertrophy was similar to the classical phenotype described by Flavigny et al. ${ }^{9}$ This study also reported two cases of sudden death at young age in a family with the Arg58Gln mutation. Although another study suggested that myosin light chain mutations cause only a benign HCM phenotype, ${ }^{11}$ the pooled data from the study of Flavigny et al..$^{9}$ and our study suggest that the Arg58Gln mutation is malignant and associated with early clinical manifestation of HCM and poor prognosis. Because of the known clinical heterogeneity of HCM, genotyping for this mutation to improve risk stratification in HCM patients is premature at the moment.

The identification of these two known mutations in our study population suggests that the codons 22 and 58 are highly susceptible to mutations ('hot spots'). Several observations support that these two mutations indeed cause HCM. First, there was a clear cosegregation of the Glu22Lys and Arg58Gln mutation with HCM in the present and in previous studies: the mutations were present in all clinically affected family members. Second, neither of these mutations was observed in control individuals of Caucasian origin (210 alleles) indicating that they are not common polymorphisms. Previous screening excluded mutations in the myosin binding protein $\mathrm{C}$ and cardiac $\alpha$-actin genes in the patients with the RLC mutations. Third, the altered residues as well as flanking sequences show strong evolutionary conservation across vertebrate species suggesting an important functional role. Both mutations are located in the amino-terminal half of the RLC containing putative phosphorylation and calcium binding domains. Thus, this mutation may alter function of the myosin molecule.

Szsesna et al..$^{23}$ investigated the effects of the Glu22Lys and Arg58Gln mutations in the RLC on $\mathrm{Ca}^{2+}$ binding, phosphorylation and secondary structural properties. They demonstrated that the Glu22Lys mutant could not be phosphorylated and showed decreased $\mathrm{Ca}^{2+}$ affinity. The Arg58Gln mutant did not bind calcium at all. Alteration of these biochemical properties of RLC could contribute to the development of HCM. It is still unclear how alterations in $\mathrm{Ca}^{2+}$ homeostasis may induce cardiac hypertrophy. The problems in understanding the functional consequences of different mutations compound the development of new treatment strategies.

\section{Conclusion}

Ventricular myosin regulatory light chain mutations are a very rare cause of $\mathrm{HCM}$ because they were present in about $1 \%$ of cases; mutations in the essential light chain are even rarer. Especially in this form of HCM, data from every single family identified help to correlate genotype and phenotype. We showed that mutations in RLC could be associated with either benign or malignant HCM phenotype. The Glu22Lys mutation was associated with late onset of clinical symptoms and good prognosis whereas the Arg58Gln mutation was associated with early clinical manifestation and premature sudden cardiac death.

\section{Acknowledgements}

We thank the families for their cooperation. We also thank $\mathrm{Dr} R$ Bergmann-Lips for supporting the clinical examination of the family members and E Szczech for technical assistance. We gratefully acknowledge Dr Damian Counsell for his helpful comments. The study was supported by Charité research grants and scholarship grants to Dr Kabaeva from Deutscher Akademischer Austauschdienst, Freunde der Charité $\mathrm{eV}$ and Deutsche Herzstiftung eV.

\section{References}

1 Richardson P, McKenna WJ, Bristow M et al: Report of the 1995 WHO/ISFK task force on the definition and classification of cardiomyopathies. Circulation 1996; 93: 841-842.

2 Maron BJ, Gardin JM, Flack JM, Gidding SS, Kurosaki TT, Bild DE: Prevalence of hypertrophic cardiomyopathy in a general population of young adults. Echocardiographic analysis of 4111 subjects in the CARDIA Study. Coronary Artery Risk Development in (Young) Adults. Circulation 1995; 92: 785 - 789. 
3 Maron BJ, Olivotto I, Spirito P et al: Epidemiology of hypertrophic cardiomyopathy-related death. Revisited in a large non-referralbased patient population. Circulation 2000; 102: 858-864.

4 Maron BJ: Ventricular arrhythmias, sudden death, and prevention in patients with Hypertrophic Cardyomyopathy. Current Cardiology Reports 2000; 2: 522-528.

5 Roberts R, Sigwart U: New concepts in Hypertrophic Cardiomyopathies, Part I. Circulation 2001; 104: 2113-2116.

6 Seidman JG, Seidman C: The genetic basis for Cardiomyopathy: from mutation identification to mechanistic paradigms. Cell 2001; 104: 557-567.

7 Vikstrom K, Leinwand LA: Contractile protein mutations and heart disease. Curr Opin Cell Biol 1996; 8: 97-105.

8 Poetter K, Jiang H, Hassanzadeh S et al: Mutations in either the essential or regulatory light chains of myosin are associated with a rare myopathy in human heart and skeletal muscle. Nat Genet 1996; 13: 63-69.

9 Flavigny J, Richard P, Isnard R et al: Identification of two novel mutations in the ventricular regulatory myosin light chain gene (MYL2) associated with familial and classical forms of hypertrophic cardiomyopathy. J Mol Med 1998; 76: 208-214.

10 Lee W-H, Hwang TH, Kimura A et al: Different expressity of a ventricular essential myosin light chain gene Ala57Gly mutation in familial hypertrophic cardiomyopathy. Am Heart J 2001; 141: $184-189$.

11 Andersen PS, Havndrup O, Bundgaard $\mathrm{H}$ et al: Myosin light chain mutations in familial hypertrophic cardiomyopathy: phenotypic presentation and frequency in Danish and South African populations. JMG 2001; 38: e43.

12 Macera MJ, Szabo P, Wadgaonkar R, Siddiqui MA, Verma RS: Localization of the gene coding for ventricular myosin regulatory light chain (MYL2) to human chromosome 12q23-q24.3. Genomics 1992; 13: 829-831.
13 Fodor WL, Darras B, Seharaseyon J, Falkenthal S, Francke U, Vanin EF: Human ventricular/slow twitch myosin alkali light chain gene characterization, sequence, and chromosomal location. J Biol Chem 1989; 264: 2143-2149.

14 Rayment I, Rypniewski WR, Schmidt-Base K et al: Three-dimensional structure of myosin subfragment-1: a molecular motor Science 1993; 261: 50-58.

15 Uyeda TQ, Abramson PD, Spudich JA: The neck region of the myosin motor domain acts as a lever arm to generate movement. Proc Natl Acad Sci USA 1996; 93: 4459-4464.

16 Trybus KM: Role of myosin light chains. J Musc Res Cell Motility 1994; 15: 587-594.

17 Morano I: Tuning the human heart molecular motors by myosin light chains. J Mol Med 1999; 77: 544-555.

18 Henry WL, DeMaria A, Gramiak R et al: Report of the American Society of Echocardiography Committee on Nomenclature and Standards in two-dimensional echocardiography. Circulation 1980; 62: 212-217.

19 McKenna WJ, Spirito P, Desnos M, Dobourg O, Komaida M: Experience from clinical genetics in hypertrophic cardiomyopathy: proposal for new diagnostic criteria in adult members of affected families. Heart 1997; 77: 130-132.

20 Henry WL, Gardin JM, Ware JH: Echocardiographic measurements in normal subjects from infancy to old age. Circulation 1980; 62: 1054-1061.

21 Beaudet AL, Tsui L-C. : A suggested Nomenclature for Designating Mutations. Hum Mutat 1993; 2: 245-224.

22 Hayashi K, Yandell DW: How sensitive is PCR-SSCP? Hum Mutat 1993; 2: $338-346$.

23 Szczesna D, Ghosh D, Li Q et al: Familial hypertrophic cardiomyopathy mutations in the regulatory light chains of myosin affect their structure, $\mathrm{Ca}^{2+}$ binding and phosphorylation. $J$ Biol Chem 2001; 276: 7086-7092. 